\title{
X-ray spectroscopy on the active ion in laser crystals
}

\author{
P. S. Miedema, ${ }^{a \dagger}$ R. Mitzner, ${ }^{a}$ S. Ganschow, ${ }^{b}$ A. Föhlisch ${ }^{a, c}$ and M. Beye ${ }^{a+}$ \\ The active ions in typical laser crystals were studied with Resonant Inelastic X-ray Scattering (RIXS) and Partial \\ Fluorescence Yield X-ray Absorption (PFY-XAS) spectroscopies as solid state model systems for dilute active centers. We \\ analyzed $\mathrm{Ti}^{3+}$ and $\mathrm{Cr}^{3+}$ in $\alpha-\mathrm{Al}_{2} \mathrm{O}_{3}: \mathrm{Ti}^{3+}$ and $\mathrm{LiCaAlF}_{6}: \mathrm{Cr}^{3+}$ respectively. The comparison of experimental data with semi- \\ empirical multiplet calculations provides insights into the electronic structure and shows how measured crystal field \\ energies are related across different spectroscopies.
}

\section{Introduction}

Active transition metal centres in a relatively inactive matrix are widespread in biology, (bio)chemical and technological applications, e.g., in enzymes, in catalysis ${ }^{1}$, or as part of photoactive molecules in solution ${ }^{2-5}$. Such diluted systems can be uniquely studied with $\mathrm{X}$-ray spectroscopies that are intrinsically element specific by addressing core levels. Here, the soft $x$-ray regime $(\sim 40-1400 \mathrm{eV})$ features the sharpest available core levels for all the elements in the Periodic Table ${ }^{6}$ and thus allows for highest energy resolution in electronic structure determination. Among the soft $\mathrm{x}$-ray spectroscopies, especially resonant inelastic x-ray scattering (RIXS) is a very powerful technique ${ }^{7-9}$ and can be viewed as a combination of x-ray absorption spectroscopy (XAS), to study unoccupied states, and $x$-ray emission spectroscopy (XES) addressing occupied valence bands.

Recently, studies of dilute molecules in liquids with RIXS (with about $0.1-1 \mathrm{~mole} / \mathrm{L})^{2,10-13}$ have been performed in addition to the more standard studies of bulk solid materials. Due to limited signal levels and thus required long-term stability, it remains difficult to perform soft X-ray RIXS at very low concentrations in liquids.

In this article we use the dopants in solid state laser crystals as dilute active centre with concentrations below (0.1 at\%) and similar to (3 at\%) generally applied for liquids. We support our RIXS measurements with multiplet simulations and compare the results to available optical data. Similarities and differences in the respectively obtained electronic structure information are discussed.

\section{Experimental and theoretical methods}

\subsection{Sample preparation}

The titanium sapphire $\alpha-\mathrm{Al}_{2} \mathrm{O}_{3}: \sim 0.1$ at $\% \mathrm{Ti}^{3+}$ (TiSa) crystal was obtained from Saint-Gobin crystals produced for usage in laser amplifiers ${ }^{14,15}$, where at\% refers to the atomic percentage of $\mathrm{Al}$ substitution by $\mathrm{Ti}$. The crystal was cut down to about 1 millimetre thickness and repolished before the measurements.

The $\mathrm{LiCaAlF}_{6}: \mathrm{Cr}^{3+}\left(\mathrm{LiCAF}: \mathrm{Cr}^{3+}\right)$ single crystal was grown using the Czochralski technique with radio-frequency induction heating. The starting material, stoichiometric or nearstoichiometric mixtures of the metal fluorides, was melted in
$40 \mathrm{ml} \mathrm{Pt}$ or Pt/Au crucibles in a gas flow of $5 \mathrm{~N}$ nitrogen with $<2$ ppm residual $\mathrm{H}_{2} \mathrm{O}$ gas content. Small amounts of $\mathrm{NH}_{4} \mathrm{HF}_{2}$ were added to the mixture in order to react with adsorbed water at elevated temperature before melting ${ }^{16,17}$. Chromium concentration in the melt was up to 3 at\% with respect to Al. Since the segregation coefficient is known to be near unity ${ }^{18}$, chromium distribution in the crystals must be expected to be homogeneous at the level of melt doping. Crystallization was initiated by pulling a seed crystal immersed into the melt upwards at simultaneous lowering of the heating power. The seed was suspended from a balance, detecting changes in the crystal weight used for the automatic diameter control. Crystals were pulled with a rate of 1.0 millimetre/h and continuously rotated to improve melt mixing and compensate for azimuthal thermal inhomogeneities caused e.g. by observation windows. The grown crystals were 18 millimetre in diameter and up to 100 millimetre long, of intense green colour and free of obvious defects like cracks, massive inclusions or similar. Prior to the measurements, the crystals were cut into millimetre thin pieces and polished.

\subsection{X-ray spectroscopy experiment}

The spectra of these samples were measured with the SolidFlexRIXS-setup featuring a Nordgren-type Grazespectrometer ${ }^{19}$ fixed in the horizontal plane at $90^{\circ}$ to the incoming beam. We used beamline U49/2-PGM- $1^{20}$ of the synchrotron Bessy-II of the Helmholtz-Zentrum Berlin. The incident energy resolution was $0.4-0.5 \mathrm{eV}$ and the spectrometer resolution was set to about $0.4 \mathrm{eV}$. The L-edge $\mathrm{X}$ ray absorption spectra of the dopants were measured by summing the total $3 \mathrm{~d}$ emission signal as a function of excitation energy often referred to as 3d-partial fluorescence yield (PFY) x-ray absorption. The PFY and RIXS experiments have been performed with linear horizontal polarized $x$-ray excitation under an incidence angle of about 40 degrees to the surface. A typical RIXS map was measured in about 3 hours. For the $\mathrm{Cr}^{3+}$, with more features to resolve, four of such measurements were added up to increase statics and no beam damage was observed on these time scales. All measurements were performed at room temperature.

\subsection{Crystal field multiplet theory}

We applied calculations in the crystal field approximation taking local electronic 3d-electron interactions into account. With Quanty ${ }^{21}$ we simulated $\mathrm{L}_{2,3}$-edge RIXS and 3d-PFY-XAS, 
where the TT-multiplet code developed by Theo Thole ${ }^{22}$, based on the Cowan code ${ }^{23}$ and additions by Butler et al. ${ }^{24}$, was used to provide the input for the Hartree-Fock Slater integrals and the atomic 3d spin-orbit coupling. These crystal field simulations were performed for the active $3 d$-metal ion, here with the electronic configurations of $[\mathrm{Ar}] 3 \mathrm{~d}^{1}$ for $\mathrm{Ti}^{3+}$ and $[\mathrm{Ar}] 3 \mathrm{~d}^{3}$ for $\mathrm{Cr}^{3+}$. Since our $\mathrm{L}_{2,3}$-edge measurements induce transitions between $2 p$ and $3 d$ orbitals, we usually refer to the relevant subset of ground state occupations: $2 p^{6} 3 d^{1}$ for $\mathrm{Ti}^{3+}$ and $2 p^{6} 3 d^{3}$ for $\mathrm{Cr}^{3+}$. In our simulations, these ions were placed in an imagined crystal field with octahedral $\left(\mathrm{O}_{h}\right)$ symmetry, splitting the energy levels of the valence $3 d$ orbitals into a threefold degenerate $t_{2 g}$ and a twofold degenerate $e_{g}$ subset. The energetic difference between electrons in those subsets is referred to as $10 \mathrm{Dq}$ and is marked in Figure 1 in red.

Since $X$-ray absorption probes the core-excited state, there can be a reaction of the surroundings to the core excitation leading to a change in the crystal field and that is why we introduced a separate crystal field parameter for the core-excited state $\left(10 D q_{E S}\right)$. We iteratively varied this parameter to agree with the experimental $x$-ray absorption spectrum. Deviations from the true XAS through using the partial fluorescence yield method have been accounted for in the simulations ${ }^{25}$. In addition, the x-ray absorption spectrum shows multiplet structures due to $2 p-3 d$ and $3 d-3 d$ interactions, which are taken into account in our simulations by the Slater integrals calculated in the Hartree-Fock approximation. In general, this approximation overestimates the Slater integrals, which can be accounted for by scaling the Hartree-Fock values with $\beta=0.8$ in accordance with Cowan's findings ${ }^{23}$ to compensate for manyelectron correlation effects not taken into account in the Hartree-Fock approximation. We point out that the CTM4XAS interface implicitly applies this scaling ${ }^{9}$ and that simulations on oxides within CTM4XAS result often in a 0.7-0.8 scaling, which would be in our case a $\beta$ of $\left(0.8^{*} 0.8=\right) 0.64$ or $\left(0.7^{*} 0.8=\right) 0.56$. This $\beta$-parameter can be reduced further to account for deviations from the purely ionic bonding through covalency effects that delocalize the $3 d$-electrons with the orbitals of neighbouring atoms leading to decreased $3 d-3 d$ electron interaction integrals.

In our simulations, we optimized both $\beta$ and $10 D q_{E S}$ to match the experimental PFY-XAS spectra. We note that $\beta$ mainly influences relative intensities and energy separations along the exciting photon energy axis as represented in PFY-XAS, with only minor influence on the spectral shape of RIXS spectra recorded at fixed photon energy for our model systems.

In the experimental samples, the $\mathrm{Cr}^{3+}$ and $\mathrm{Ti}^{3+}$ dopant ions are distributed randomly over various sites in the crystal resulting in nearly random relative orientations. Therefore isotropic PFY-XAS and RIXS simulations were applied, even though, as mentioned in section 2.2, the $x$-ray spectroscopy experiments were performed with linear horizontal polarized $x$-ray excitation under an incidence angle of about 40 degrees to the surface and the detector placed in the horizontal scattering plane perpendicular to the beam. All states that were within $0.02 \mathrm{eV}$ from the observed initial ground state were taken into account in the self-consistent field cycle for the ground state electronic structure. The actual RIXS and PFY-XAS simulations were performed on only the (four-fold-degenerate for both $\mathrm{Ti}^{3+}$ and $\mathrm{Cr}^{3+}$ ) ground state. All simulations were Lorentzian broadened with $0.5 \mathrm{eV}$.
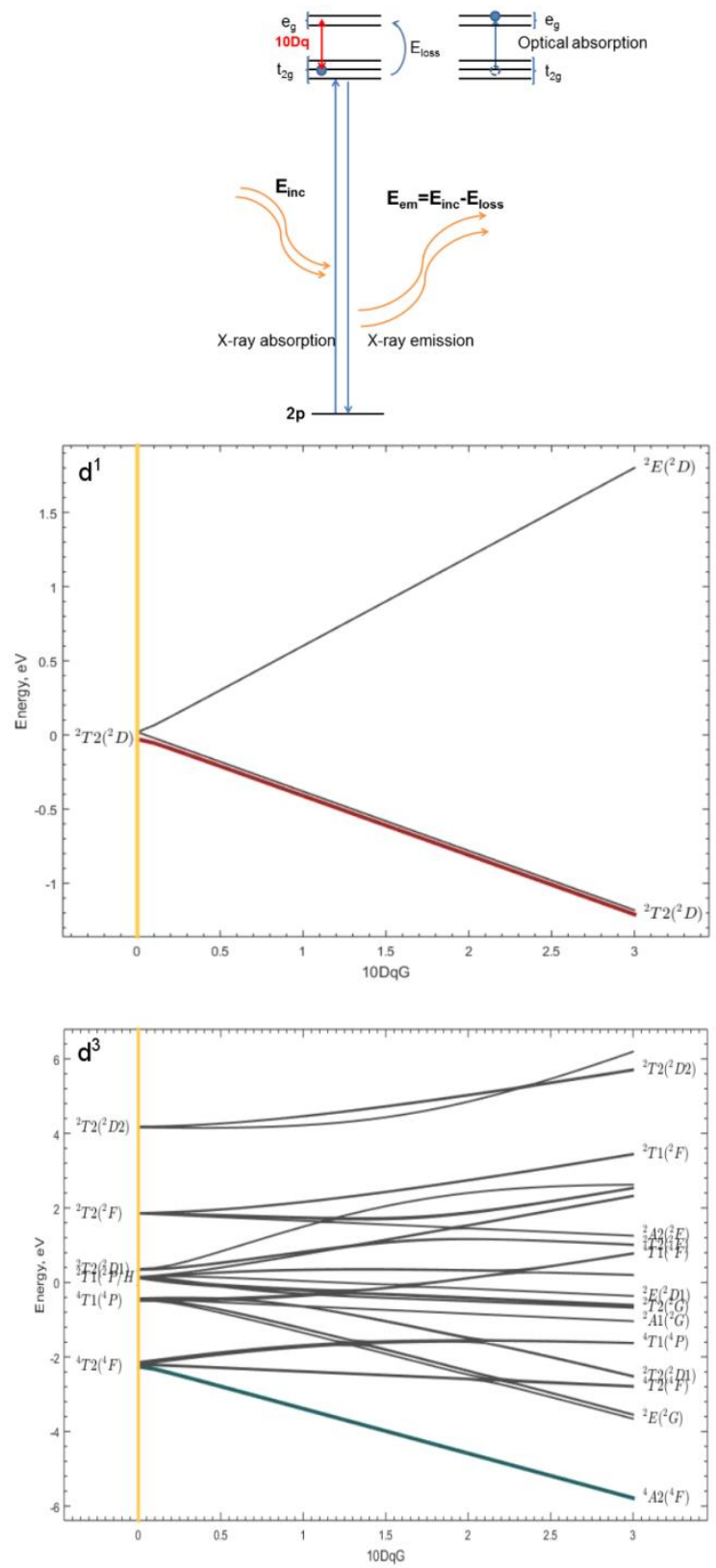

Figure 1. The RIXS process (top) and Tanabe-Sugano diagrams constructed with CTM4DOC ${ }^{26}$ for $\mathrm{Ti}^{3+}\left(\mathrm{d}^{1}\right.$, middle panel) and $\mathrm{Cr}^{3+}\left(\mathrm{d}^{3}\right.$, lowest panel) providing possible optical $3 d-3 d$ excitations. Incoming $X$-rays with the energy $E_{\text {inc }}$ are absorbed by exciting a $2 p$ electron to an empty $3 d$ level. Radiative decays fill the $2 p$ core level while emitting $x$-rays with energy $E_{\text {em }}$. The energy difference $\left(E_{\text {loss }}\right)$ between the $x$-ray absorption ( $\left.E_{\text {inc }}\right)$ and the $x$-ray emission $\left(E_{e m}\right)$ is connected to an excitation in the system, for example between $t_{2 g}$ and $e_{g}$ states. The crystal field energy $10 \mathrm{Dq}$ is marked as the transition of an electron from a $t_{2 g}$ orbital to an $e_{g}$ orbital. A $3 d-3 d$ excitation measured with optical absorption in UV/vis spectroscopy (right) directly corresponds to the energy loss in RIXS. Excited 3d-3d states that can be reached through absorption / RIXS energy loss for $\mathrm{Ti}^{3+}$ and $\mathrm{Cr}^{3+}$ are presented in the Tanabe-Sugano diagrams in the middle and lowest panel where colored red and blue lines represent the ground state. The gerade symbols for $\mathrm{O}_{\mathrm{h}}$ symmetry are omitted from the state assignments. 


\subsection{Linking 3d-3d excitations in RIXS with UV/vis spectroscopy}

For a transition metal in an $\mathrm{O}_{\mathrm{h}}$ crystal field, typically the excitation from a $t_{2 g}$ orbital into an $e_{g}$ orbital is one of the lowest possible electronic excitations visible, usually located in the UV/vis energy region and yields the value of $10 \mathrm{Dq}$ as for the single $3 d$-electron in $\mathrm{Ti}^{3+}$. One may expect as well intra- $\mathrm{t}_{2 \mathrm{~s}}$ 3d-3d excitations (see Tanabe-Sugano diagram for a singly occupied $3 d$ orbital $\left(d^{1}\right)$ in Figure 1 ) due to $3 d$ spin orbit interaction, but these appear rather weak and are commonly not observed experimentally within typical resolutions because the $3 \mathrm{~d}$ spin-orbit coupling is of the order of $50 \mathrm{meV}$. In general though, dipole transitions between the orbitals of the same subshell are forbidden, following the selection rules $(\Delta \mathrm{L}= \pm 1, \Delta \mathrm{S}=0)$. Nevertheless, $3 \mathrm{~d}-3 \mathrm{~d}$ excitations often weakly appear in UV/Vis spectra because of phonon coupling for octahedral systems or by removal of the g/u character in noncentrosymmetric sites (in non-octahedral systems). Therefore the octahedral $10 \mathrm{Dq}$ can be extracted, albeit with potential modification through the coupling.

In contrast to optical absorption, RIXS involves two dipole transitions - absorption followed by emission - as in resonantly enhanced Raman spectroscopy. Excitations in the material are encoded in the energetic difference between the two (see Fig. 1). In such a Raman process, 3d-3d excitations are dipole-allowed and RIXS can for example directly measure $10 \mathrm{Dq}$ in the ground state without the necessity to couple to phonons.

\section{Results and discussion}

We will first describe the experimental and theoretical $\mathrm{Ti}_{2,3^{-}}$ edge RIXS obtained for TiSa and $\mathrm{Cr}_{2,3}$-edge RIXS obtained for LiCAF: $\mathrm{Cr}^{3+}$. After that we discuss the PFY-XAS results for both compounds.

\subsection{Ti $\mathrm{L}_{2,3}$-edge RIXS of TiSa}

Following the considerations in section 2.4, the first energy loss feature in the RIXS spectra of $\mathrm{Ti}^{3+}$ that is sufficiently separated from the elastic emission should directly yield 10Dq. We used our measurements to fix this $10 \mathrm{Dq}_{\text {initial }}$ as the crystal field splitting of the ground state and simulated the RIXS spectra accordingly. UV/vis finds a 10Dq of $2.23 \mathrm{eV}$ for TiSa ${ }^{14}$. The $10 \mathrm{Dq}_{\text {initial }}$ of $2.2 \mathrm{eV}$ found in the RIXS experiment agrees well within the measurement resolution. In the $x$-ray absorption process of $\mathrm{Ti}^{3+}$, a $2 p$ electron is excited into the unoccupied $3 d$ manifold $\left(2 p^{6} 3 d^{1} \rightarrow 2 p^{5} 3 d^{2}\right)$, thereby allowing for $3 d-3 d$ (and $2 p-3 d$ ) interactions in the core-excited state. Multiple structures appear in the spectrum as function of photon energy (e.g., see Figure 6 and Figure 7 later on) and the shape of the $\mathrm{x}$-ray absorption spectrum depends strongly on $10 \mathrm{Dq}^{27,28}$. However, in the initial and final state of RIXS, the filled $2 p$ shell and the single $3 d$ electron do not allow for such interactions. Only one certain $3 d-3 d$ excitation remains for a $3 d^{1}$ system within our experimental resolution (ignoring the possible intra- $t_{2 g}$ excitations at very low energy as seen in the Tanabe-Sugano diagram of $d^{1}$ in Figure 1): the $t_{2 g}-e_{g}$ excitation remains visible in the experimental (and simulated) spectrum which directly corresponds to the energetic value of 10Dq.
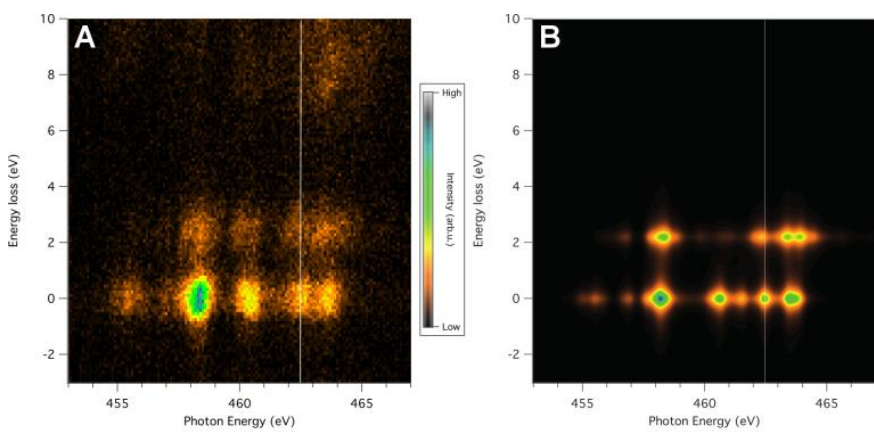

Figure 2. A) Experimental Ti $\mathrm{L}_{2,3}$-edge RIXS map of TiSa with energy loss (vertical axis) versus exciting photon energy (horizontal axis) B) Ti $\mathrm{L}_{2,3}$-edge RIXS map calculated with the parameters $10 \mathrm{Dq}_{\text {initial }}=2.2 \mathrm{eV}, 10 \mathrm{Dq_{ES }}=1.98 \mathrm{eV}$ and $\beta=0.55$. The simulation results are shifted in exciting photon energy by $458.7 \mathrm{eV}$. The white vertical lines mark the slices shown in Figure 3.

Figure 2 shows the comparison of the experimental and simulated $\mathrm{Ti}_{2,3}$-edge RIXS maps of TiSa while Figure 3 shows one vertical slice of these RIXS maps and the energies match but the intensity and broadening is different. Features above an energy loss of $5 \mathrm{eV}$ only appear in the experimental spectrum and can be related to charge transfer excitations, which are not taken into account in our simulations. They could be reproduced by explicitly accounting for charge transfer between the metal ion and the ligands ${ }^{8,9}$ but do not contain further information on the optical activity of the ion.

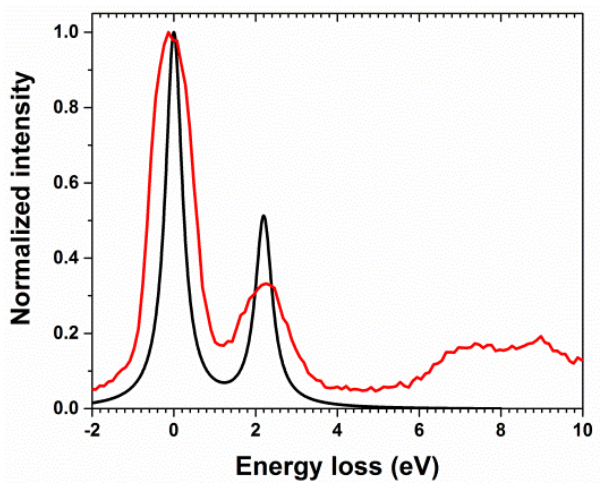

Figure 3. RIXS slices of the RIXS maps in Figure 2 at an excitation energy of $462.5 \mathrm{eV}$ as function of energy loss from the simulated $T_{i} L_{2,3}$ RIXS map in Figure $2 B$ (black) and the experimental RIXS taken from Figure 2A (red).

\section{2 $\mathrm{Cr} \mathrm{L}_{2,3}$-edge RIXS of LiCAF: $\mathrm{Cr}^{3+}$}

Figure 4 shows the experimental and simulated $\mathrm{Cr} \mathrm{L}_{2,3} \mathrm{RIXS}$ maps of LiCAF: $\mathrm{Cr}^{3+}$. More features are visible as compared to the $\mathrm{Ti}^{3+}$ maps in Figure 2. Now, multiplet interactions of $\mathrm{Cr}^{3+}$ $\left(3 d^{3}\right)$ in both the ground state ( $3 d-3 d$ interactions) as well as in the $x$-ray excited state $(2 p-3 d$ and additional $3 d-3 d$ interactions) appear. The first resolved RIXS transition can be again be identified with a $t_{2 g}$ - $e_{g}$ transition with energy of $10 \mathrm{Dq}$ (see Figure 1 lowest panel: ${ }^{2} E_{g}$ and ${ }^{4} T_{1 g}$ are the first $3 d-3 d$ excitations which refer to $t_{2 g}-e_{g}$ excitations). 

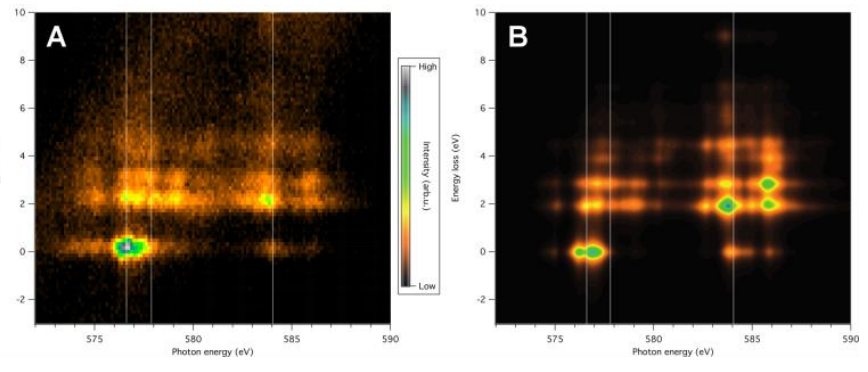

Figure 4. A) Experimental $\mathrm{Cr} \mathrm{L}_{2,3}$-edge RIXS map of $\mathrm{LiCaAlF}_{6}: \mathrm{Cr}^{3+}$ with energy loss (vertical axis) versus exciting photon energy (horizontal axis). B) $\mathrm{Cr}_{2,3}$-edge RIXS map calculated with the parameters $10 \mathrm{Dq}_{\mathrm{initial}}=1.9 \mathrm{eV}, 10 \mathrm{Dq} \mathrm{Es}_{\mathrm{ES}}=1.71 \mathrm{eV}$ and $\beta=0.75$. The simulated results are shifted in photon energy by $578 \mathrm{eV}$. The white vertical lines mark the slices shown in Figure 5.

In the experimental RIXS we find a value for 10Dq of $1.9 \mathrm{eV}$ in agreement with other data ${ }^{15,29,30}$ : This value is in between experimental and theoretical optical data of $1.83 \mathrm{eV}$ and 2.02 eV for excitations from the ground initial state ${ }^{4} A_{2 g}$ to the ${ }^{2} E_{g}$ and ${ }^{4} T_{1 g}$ state (see as well the Tanabe-Sugano diagram for $d^{3}$ in Figure 1) respectively ${ }^{29}$. From other literature on optical studies of LiCAF:Cr ${ }^{3+}$ it is known that $10 \mathrm{Dq}_{\text {initial }} \sim 2.0 \mathrm{eV}^{15,30}$.

Figure 5 provides a more detailed comparison of the experiment and the theoretical simulation by RIXS slices taken from the simulated and experimental $\mathrm{Cr}_{2,3}$-edge RIXS maps of LiCAF: $\mathrm{Cr}^{3+}$ and of the simulation of $\mathrm{Cr}^{3+}$ with $10 \mathrm{Dq}_{\text {initial }}=1.9 \mathrm{eV}$, $10 D q_{E S}=1.71 \mathrm{eV}$ and $\beta=0.75$.

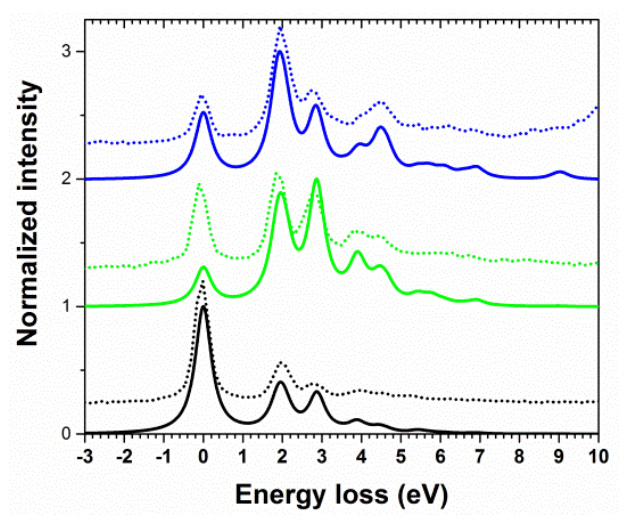

Figure 5. RIXS slices at photon energies of $576.8 \mathrm{eV}$ (black), $577.9 \mathrm{eV}$ (green) and 584.2 $\mathrm{eV}$ (blue) as function of energy loss from the simulated $\mathrm{Cr}_{2,3} \mathrm{RIXS}$ map in Figure $4 \mathrm{~B}$ (solid lines) and from the experimental $\mathrm{Cr}_{2,3}$ RIXS map in Figure 4A (dotted lines).

In contrast to TiSa, the various multiplet interactions yield energy loss features that extend up to about $10 \mathrm{eV}$. Only at about 9-10 eV, broader features appear in the experiment (e.g., the blue dotted line) due to charge transfer excitations. The simulations reproduce all other features. For some RIXS slices the relative intensity of the elastic line differs between experiment and simulation (compare the green lines), but the inelastic features can be reproduced both in relative intensity as well as peak position.

In order to match the full RIXS map with structures in both the energy loss features as well as along the exciting photon energy axis, we had to introduce different crystal field parameters for the ground and the core excited states. With the ground state $10 \mathrm{Dq}$ fixed by the first excitation in the RIXS spectra, we now analyze in the PFY-XAS how 10Dq of the coreexcited state $\left(10 \mathrm{Dq} \mathrm{ES}_{\mathrm{ES}}\right)$ is determined.

\subsection{TiSa PFY-XAS}

Figure 6 shows the Ti $\mathrm{L}_{2,3}$-edge PFY-XAS of TiSa (blue line) in comparison to some simulations of $\mathrm{a} \mathrm{Ti}^{3+}$ ion in $\mathrm{O}_{\mathrm{h}}$ symmetry using different values for $10 \mathrm{Dq}$. It becomes apparent that using the same crystal field parameters for RIXS and XAS $\left(10 \mathrm{Dq} \mathrm{q}_{\text {initial }}=10 \mathrm{Dq}_{\mathrm{ES}}=2.2 \mathrm{eV}\right)$ does not agree well enough yet with the measured absorption spectrum. Especially around 461.5 $\mathrm{eV}$ the simulation yields intensity in an additional feature, which is not prominent in the experiment. Best agreement with the experimental data is found for $10 \mathrm{Dq}=1.98 \mathrm{eV}$, a $90 \%$ scaling of the ground state value (red line, Figure 6). A deviating $10 \mathrm{Dq}_{\text {initial }}$ of $2.2 \mathrm{eV}$ to match the RIXS data above, while keeping $10 \mathrm{Dq}_{\mathrm{ES}}=1.98 \mathrm{eV}$ (green line, Figure 6) does not influence the absorption spectrum (compare the red and the green lines in Figure 6: these are equivalent).

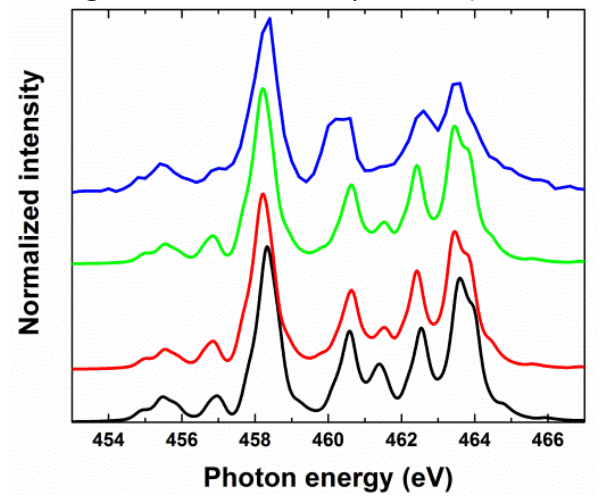

Figure 6. Ti $\mathrm{L}_{2,3}$-edge partial fluorescence yield (PFY) $\mathrm{x}$-ray absorption spectrum (XAS) of $\mathrm{Ti}^{3+}: \alpha-\mathrm{Al}_{2} \mathrm{O}_{3}$ ( $\mathrm{TiSa}$, top, blue) compared to simulations of $\mathrm{Ti}^{3+}$ in $\mathrm{O}_{\mathrm{h}}$ symmetry with $10 \mathrm{Dq} q_{\text {initial }}=10 \mathrm{Dq}_{\mathrm{ES}}=2.2 \mathrm{eV}$ and $\beta=0.55$ (black), $10 \mathrm{Dq} q_{\text {initial }}=10 \mathrm{Dq} \mathrm{ES}_{\mathrm{ES}}=1.98 \mathrm{eV}$ and $\beta=0.55$ (red), and $10 \mathrm{Dq}_{\text {initial }}=2.2 \mathrm{eV}, 10 \mathrm{Dq} \mathrm{Es}_{\mathrm{E}}=1.98 \mathrm{eV}$ and $\beta=0.55$ (green). All simulated spectra are broadened with $0.5 \mathrm{eV}$ Lorentzian broadening.

We have also verified how distortions from octahedral symmetry affect the PFY-XAS, since tetragonal or trigonal distortions from $\mathrm{O}_{\mathrm{h}}$ symmetry to $\mathrm{D}_{4 \mathrm{~h}}$ (or $\mathrm{D}_{3 \mathrm{~d}}$ ) symmetry appear in $\mathrm{Ti} \mathrm{L}_{2,3}$-edge $\mathrm{x}$-ray absorption spectra of $\mathrm{Ti}^{4+}$-containing minerals ${ }^{31}$ and a trigonal distortion is reported for $\mathrm{TiSa}^{32,33}$. We simulate an analogous situation by introducing additional parameters on top of 10Dq: We use an additional splitting $\Delta_{1}$ between the $t_{2 g}$ orbital energies and $\Delta_{2}$ between the $e_{g}$ orbital energies. Although the fundamental parameters for particular $D_{4 h}$ or $D_{3 d}$ symmetry distortions are $D s, D t$ or $D \sigma, D \tau$, we just aimed to simulate the resulting effect on the splitting of the 3d-orbitals, which directly encodes in the $\Delta_{1}$ and $\Delta_{2}$ parameters. We noticed that the agreement between simulations and experiment becomes worse with strong distortion. For small distortion, the spectral changes are below our experimental resolution. Therefore it is concluded that within the experimental accuracy of our soft $x$-ray $L_{2,3}$-edge PFY-XAS and RIXS experiments, the $\mathrm{Ti}^{3+}$ can be regarded as being in an $\mathrm{O}_{\mathrm{h}}$ symmetric surrounding.

Using the Hartree-Fock Slater integral scaling parameter $\beta$ to mimic the degree of covalency and match relative peak 
positions and intensities, best agreement for TiSa is found with $\beta=0.55(+/-0.05)$ and all presented simulations for $\mathrm{Ti}^{3+}$ use this value.

\subsection{LICAF:Cr3+ PFY-XAS}

In Figure 7 the same analysis is shown for the $\mathrm{Cr} \mathrm{L}_{2,3}$-edge PFYXAS of LiCAF: $\mathrm{Cr}^{3+}$. With the simulations we demonstrate, also here, optimal agreement for a $90 \%$ scaling of the ground state value $10 \mathrm{Dq}_{\mathrm{ES}}=1.71 \mathrm{eV}=0.9 * 10 \mathrm{Dq}$ initial $_{\text {creating best agreement }}$ around $579 \mathrm{eV}$ and $584 \mathrm{eV}$ (compare with black vertical lines). We further observed again no indication of a distorted symmetry from $\mathrm{O}_{\mathrm{h}}$ Thus again, best match between simulation and experiment is found for an undistorted $\mathrm{O}_{\mathrm{h}}$ symmetry within our experimental accuracy. A difference between the two crystals is identified in optimizing the $\beta$ parameter: best agreement is achieved for LiCAF: $\mathrm{Cr}^{3+}$ with a value of 0.75 as opposed to the 0.55 found for TiSa. This confirms the intuitively expected larger ionic character of fluorine ligands as compared to more covalent oxygen ligands.

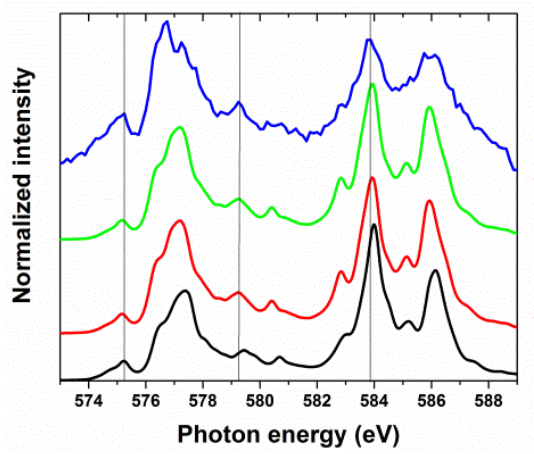

Figure 7. $\mathrm{Cr}_{2,3}$-edge partial fluorescence yield (PFY) $\mathrm{x}$-ray absorption spectra (XAS) of LiCaAlF $: \mathrm{Cr}^{3+}$ (top, blue) compared to simulations with $10 \mathrm{Dq}_{\text {initial }}=10 \mathrm{Dq}_{\mathrm{ES}}=1.9 \mathrm{eV}$ and $\beta=0.75$ (black), $10 \mathrm{Dq} q_{\text {initial }}=10 \mathrm{Dq}_{\mathrm{ES}}=1.71 \mathrm{eV}$ and $\beta=0.75$ (red), $10 \mathrm{Dq} \mathrm{q}_{\text {initial }}=1.9$, and $10 \mathrm{Dq}_{\text {initial }}=1.9,10 \mathrm{Dq}_{\mathrm{ES}}=1.71 \mathrm{eV}$ and $\beta=0.55$ (green). All simulated spectra are broadened with $0.5 \mathrm{eV}$ Lorentzian broadening. Vertical black lines mark peaks of the experimental PFY-XAS.

\subsection{Discussion on symmetry distortion sensitivity}

Based on our experimental data and theoretical analysis, it seems that soft $X$-ray $L_{2,3}$-edge (PFY-)XAS is thus less sensitive to local symmetry distortions than metal K-edge $\mathrm{x}$-ray absorption natural linear dichroism (XNLD) or hard X-ray preedge XAS/RIXS as seen for example for iron systems ${ }^{34-36}$. For a deeper analysis of distortions, polarization-dependent XAS, e.g., $X N_{L D}{ }^{37}$ and hard $x$-ray K-edge RIXS ${ }^{34}$ are more sensitive than the soft $x$-ray $L_{2,3}$-edge RIXS experiments provided here, since those techniques have been shown to be able to identify different types of distortion ${ }^{34}$. However XNLD and 1s $2 p$ RIXS remain excited-state probes, so without further data it would be unclear if the observed distortion comes from the actual probe $x$-ray excitation or if the distortion is already present in the ground state (before $x$-ray absorption), while the soft X-ray $\mathrm{L}_{2,3}$-edge RIXS is capable of finding the ground state properties and the optical excited states without possible $\mathrm{x}$-ray induced changes. With better energy resolution for $\mathrm{L}_{2,3}$-edge RIXS one might actually be able to provide more information on symmetry distortion, since additional $3 d-3 d$ excitations might become visible with better energy resolution. On the other hand, for the $\mathrm{L}_{2,3}$-edge PFY-XAS, the appearance of many multiplet features makes it less sensitive to small symmetry distortions, while large symmetry distortions (for our simulations $\Delta_{1}$ or $\Delta_{2}>0.5 \mathrm{eV}$ ) can be picked up by both $\mathrm{L}_{2,3^{-}}$ edge PFY-XAS and RIXS as we observed in our simulations (not presented here).

\subsection{Discussion on $10 D q_{\text {initial }}$ vs $10 D q_{E S}$}

The trend of observing a lower $10 D q_{\mathrm{ES}}$ in XAS than in the ground state $\left(10 D q_{\text {initial }}\right)$ has previously been observed ${ }^{38}$, for example in a comparison of UV/vis, TDDFT simulations and $\mathrm{L}_{2,3}$-edge RIXS on cobalt compounds ${ }^{39,40}$ and this observation is rationalized by a reaction of the ligand field to the excitation of an electron from a local $2 p$ to a more delocalized $3 d$ state. In addition to the previous results we actually quantified the decreased $10 \mathrm{Dq} \mathrm{FS}_{\mathrm{ES}}$ in XAS as compared to $10 \mathrm{Dq} \mathrm{q}_{\text {initial }}$ for two different systems: Using a scaling of $90 \%$, we propose here a general rule of thumb to connect ground state $10 \mathrm{Dq}_{\text {initial }}$ as relevant for optical excitations with values obtained from element specific X-ray absorption spectroscopy.

\subsection{Discussion on RIXS maps versus RIXS cuts}

For the relatively simple diluted cases in this manuscript, a single RIXS cut would have been sufficient to obtain $10 \mathrm{Dq} q_{\text {initial. }}$. For more complex diluted systems (e.g., with lower symmetry or for systems with more 3d-electrons or with possible beam damage quickly arising) one may obtain a RIXS map to identify which $3 d-3 d$ excitations link to $10 \mathrm{Dq}$ and which to lower symmetry crystal field parameters. Especially consider the possibility that features may only appear at particular excitation energies. This might disturb the analysis when only a single RIXS cut has been measured at the wrong excitation energy. Therefore we recommend the use of RIXS maps in a first step to identify well-chosen excitation energies for RIXS cuts with more statistics.

\section{Conclusions}

In summary, we demonstrated the power of RIXS to study fundamental electronic structure parameters of dilute active metal ions embedded in an inactive matrix. By comparison with detailed calculations using the Quanty code, we find that $\mathrm{O}_{\mathrm{h}}$ symmetry is a good approximation for the embedding crystal and validate the larger influence of covalency for oxygen ligands as compared to more ionic fluorine systems.

We further stress that RIXS is a superior probe for ground state electronic parameters as compared to XAS and XNLD, where $x$ ray excited parameters are observed or even optical spectroscopy. With the energy loss in RIXS one measures optical transitions (albeit with Raman selection rules) and one can directly relate to the ground state properties of the material relevant for its biological, chemical or photo-activity. As opposed to optical spectroscopy though, RIXS is elementselective and can thus disentangle influences from different active ions in more complex compounds. We further demonstrate that in contrast to RIXS, XAS measures an altered 
crystal field parameter due to the reaction of the system to the core hole, although we also find that for both studied system this parameter can be scaled back to the ground state value.

\section{Acknowledgements}

We thank T. Blume for technical support of the SolidFlexRIXSchamber and HZB for beamtime allocation. PSM thanks Prof. M. Haverkort for support with the Quanty simulations. The RIXS simulations of Quanty were performed on the Maxwellcluster at DESY. MB acknowledges financial support from the Volkswagenstiftung. We further thank Christiane Förster for cutting the crystals prior to the experiment.

\section{Notes and references}

R. A. van Santen, P. W. N. M. van Leeuwen, J. A. Moulijn and B. A. Averill, Eds., Catalysis: An Integrated Approach, Elsevier Science B.V., Amsterdam, The Netherlands, 2nd, revis edn., 2000. K. Kunnus, W. Zhang, M. G. Delcey, R. V Pinjari, P. S. Miedema, S. Schreck, W. Quevedo, H. Schroeder, A. Föhlisch, K. J. Gaffney, M. Lundberg, M. Odelius and P. Wernet, JPCB, 2016, 120, 7182-7194. M. Chergui, Acc. Chem. Res., 2015, 48, 801-808. W. Zhang and K. J. Gaffney, Acc. Chem. Res., 2015, 48, 1140-1148. 2000, 29, 419-427. L. J. P. Ament, M. van Veenendaal, T. P. Devereaux, J. P. Hil and J. van den Brink, Rev. Mod. Phys., 2011, 83, 705-767.

A. Kotani, Eur. Phys. J. B, 2005, 47, 3-27.

F. de Groot and A. Kotani, Core Level Spectroscopy of Solids, 2008

K. Kunnus, I. Rajkovic, S. Schreck, W. Quevedo, S. Eckert, M. Beye, E. Suljoti, C. Weniger, C. Kalus, S. Grübel, M. Scholz, D. Nordlund, W. Zhang, R. W. Hartsock, K. J. Gaffney, W. F. Schlotter, J. J. Turner, B. Kennedy, F. Hennies, S. Techert, P. Wernet and A. Föhlisch, Rev. Sci. Instrum., 2012, 83, 123109.

11 S. Schreck, A. Pietzsch, K. Kunnus, B. Kennedy, W. Quevedo, P. S. Miedema, P. Wernet and A. Föhlisch, Struct. Dyn., 2014, 1, 54901.

12 L. Weinhardt, E. Ertan, M. lannuzzi, M. Weigand, O. Fuchs, M. Bär, M. Blum, J. D. Denlinger, W. Yang, E. Umbach, M. Odelius and C. Heske, Phys. Chem. Chem. Phys., 2015, 17 27145-53.

13 L. Weinhardt, M. Blum, O. Fuchs, A. Benkert, F. Meyer, M. Bär, J. D. Denlinger, W. Yang, F. Reinert and C. Heske, J. Electron Spectros. Relat. Phenomena, 2013, 188, 111-120.

14 P. F. Moulton, J. Opt. Soc. Am. B, 1986, 3, 125-133.

15 W. T. Silfvast, Laser Fundamentals, Cambridge University Press, Cambridge, 2nd edn., 2004 D. Klimm and P. Reiche, Cryst. Res. Technol., 1998, 33, 409416. D. Klimm and P. Reiche, J. Cryst. Growth, 2000, 210, 683-
693. R. F. Belt and R. Uhrin, J. Cryst. Growth, 1991, 109, 334339.

J. Nordgren, G. Bray, S. Cramm, R. Nyholm, J.-E. Rubensson and N. Wassdahl, Rev. Sci. Instrum., 1989, 60, 1690.

T. Kachel, J. large-scale Res. Facil., 2016, 2, A72. M. W. Haverkort, J. Phys. Conf. Ser., 2016, 712, 12001. B. T. Thole, R. D. Cowan, G. A. Sawatzky, J. Fink and J. C. Fuggle, Phys. Rev. B, 1985, 31, 6856-6858.

R. D. Cowan, The Theory of Atomic Structure and Spectra, University of California Press, Berkeley and Los Angeles, California, 1981.

P. H. Butler, Point Group Symmetry Applications - Methods and Tables, Plenum Press, New York, 1981.

P. S. Miedema, P. Wernet and A. Föhlisch, PRA, 2014, 89, 52507.

M. U. Delgado-Jaime, K. Zhang, J. Vura-Weis and F. M. F. de Groot, J. Synchrotron Radiat., 2016, 23, 1264-1271.

F. M. F. de Groot, J. C. Fuggle, B. T. Thole and G. A. Sawatzky, Phys. Rev. B, 1990, 42, 5459-5468.

G. van der Laan and I. W. Kirkman, J. Phys. Condens. Matter, 1992, 4, 4189-4204.

L.-R. Yang, C.-F. Wei, Y. Mei and W.-C. Zheng, J. Fluor. Chem., 2016, 189, 39-42.

U. Demirbas, M. Schmalz, B. Sumpf, G. Erbert, G. S. Petrich, L. a Kolodziejski, J. G. Fujimoto, F. X. Kärtner and A. Leitenstorfer, Opt. Express, 2011, 19, 20444-20461. F. M. F. de Groot, M. O. Figueiredo, M. J. Basto, M. Abbate, H. Petersen and J. C. Fuggle, Phys Chem Miner., 1992, 19, 140-147.

E. Gaudry, D. Cabaret, P. Sainctavit, C. Brouder, F. Mauri, J. Goulon and A. Rogalev, J. Phys. Condens. Matter, 2005, 17, 5467-5480.

D. S. McClure, J. Chem. Phys., 1962, 36, 2757.

M. Hunault, V. Vercamer, M. W. Haverkort, M.-A. Arrio, C. Brouder, G. Calas and A. Juhin, J. Phys. Conf. Ser., 2016, 712, 12005.

V. Vercamer, M. O. J. Y. Hunault, G. Lelong, M. W. Haverkort, G. Calas, Y. Arai, H. Hijiya, L. Paulatto, C. Brouder, M. Arrio and A. Juhin, Phys. Rev. B, 2016, 94, 245115.

T. E. Westre, P. Kennepohl, J. G. Dewitt, B. Hedman, K. O. Hodgson and E. I. Solomon, J. Am. Chem. Soc., 1997, 119, 6297-6314.

A. Juhin, F. de Groot, G. Vankó, M. Calandra and C. Brouder, Phys. Rev. B, 2010, 81, 115115.

S. P. Cramer, F. M. F. de Groot, Y. Ma, C. T. Chen, F. Sette, C. A. Kipke, D. M. Eichhorn, M. K. Chan, W. H. Armstrong, E. Libby, G. Christou, S. Brooker, V. McKee, O. C. Mullins and J. C. Fuggle, JACS, 1991, 113, 7937-7940.

M. M. van Schooneveld, R. W. Gosselink, T. M. Eggenhuisen, M. Al Samarai, C. Monney, K. J. Zhou, T. Schmitt and F. M. F. De Groot, Angew. Chemie (Int. ed.), 2013, 52, 1170-1174.

M. M. van Schooneveld, A. Juhin, C. Campos-Cuerva, T. Schmitt and F. M. F. de Groot, J. Phys. Chem. C, 2013, 117, 14398-14407. 